\title{
Die Datenmodellstandardisierung und ihre Auswirkungen auf RFID-Bibliotheken
}

\author{
Christian Kern
}

\section{Ausgangslage}

Bereits 2002, als sich die ersten Bibliotheken für den Einsatz von RFID-Systemen entschlossen, wurde auf vorhandene Standards für die Entwicklung der Selbstverbuchung und Mediensicherung zurückgegriffen. Ohne diese wäre der heutige Markt für RFIDSysteme in Bibliotheken nicht entstanden. Dabei handelte es sich, erstens, um das SIP2Protokoll (Standard Interchange Protocol $2^{1}$ ) für die Verbindung zwischen RFID-System und Bibliotheks-Management-System (LMS, Library Management System). Zweitens war der RFID-Standard ISO $15693^{2}$ ausschlaggebend, welcher die so genannte Luftschnittstelle zwischen RFID-Etiketten und Lesegeräten beschrieb und aus dem SmartcardBereich stammte. Dieser Standard war die wichtigste technische Voraussetzung für die Entwicklung des Massenmarktes für RFID-Etiketten. Weitere Standards und Regelwerke existierten zur Sendeleistung und CE-Zeichen, und schliesslich gab es noch die so genannte ISIL-Nummer (International Standard Identifier for Libraries). Als eindeutige Bibliothekskennzeichnung wird sie für die Zuordnung der Medien zu einer spezifischen Bibliothek genutzt. Die jeweiligen Mediennummern einer Bibliothek zusammen mit der ISIL ermöglichen weltweit eine eindeutige Kennzeichnung der Medien.

Die jüngste Entwicklung in diesem Reigen der Standards - und dies ist auch ein Novum innerhalb anderer RFID-Anwendungen (Logistik, Lagerhaltung, Prozesskontrolle usw.) - ist die Festlegung eines Datenmodells für die RFID-Etiketten in ISO 28560, d. h. ihres Dateninhaltes und dessen Schreibweise im Speicher ${ }^{3}$ eines RFID-Chips. Neu ist daran, dass nicht nur die Art der Kommunikation (im übertragenen Sinne auf die Sprache: wie werden Buchstaben ausgetauscht), sondern auch deren Inhalt (Festlegung der Sprache, Wörter und Inhalte) definiert werden. Im Folgenden werden der heutige Status, die bereits sichtbaren und zukünftigen Auswirkungen der Datenmodellstandardisierung beschrieben.

\section{Was ist ein RFID-Datenmodell für Bibliotheken und wie wirkt es sich aus?}

Ein Beispiel verdeutlicht die Konsequenzen fehlender Standards: Im Jahre 2002 wurden in der Rockefeller Library in New York proprietäre Chips eines bestimmten Lieferanten verwendet. Nach einiger Zeit konnten keine Etiketten mehr nachgeliefert werden, weil der Chipproduzent keine kompatiblen Halbleiter der ersten Generation mehr herstellte.

1 Entwickelt von 3M. Später ergänzt durch ein Protokoll der NISO (National Information Standards Organization)

2 Später in ISO 18000-3.1 integriert

3 RFID-Chips sind einfache, über Funk ansprechbare Datenträger (sie enthalten ein EEPROM). Die Daten können auf Speicherseiten abgelegt werden. 
Folglich mussten die bestehenden RFID-Etiketten aus den Büchern herausgetrennt und durch neue ersetzt werden. Dies bedeutete bei 100`000 Medien nicht nur einen grossen Arbeitsaufwand, sondern führte auch zur Beschädigung der Medien durch das Entfernen der veralteten Etiketten. Dieser Vorfall fand nur wenig Beachtung in den Fachzeitschriften, da dieses Problem als Einzelfall betrachtet wurde. Nach diesem »worst case« wurden jedoch in allen folgenden Installationen in den USA und Europa Chips nach ISO 15693 (heute ISO 18000-3.1) eingesetzt. Dadurch konnte auf der Chipebene eine langfristige Versorgung der Bibliotheken sichergestellt werden. Nun war es zumindest theoretisch möglich, die Chips verschiedener Hersteller, die alle diesem Standard unterlagen, innerhalb einer Bibliothek einzusetzen.

Die Standardisierung eines Datenmodells wirkt sich auf einer weiteren Ebene aus. Sie führt nicht nur zu einer Austauschbarkeit der Chips, sondern sämtlicher RFID-Geräte, wie Sicherungsgates, Verbuchungsstationen usw...

Doch betrachten wir zunächst die Ausgangslage. RFID-Bibliotheken nutzen heute, weltweit, mehrheitlich proprietäre, d. h. nicht standardisierte Datenmodelle. Damit ist ein sicherer Betrieb der Bibliothek durchaus gewährleistet - solange der Lieferant nicht gewechselt wird.

Ein proprietäres Datenmodell bedeutet, dass der Lieferant die Daten so in die Etiketten schreibt, dass diese nur von ihm selbst entschlüsselt werden können. Im übertragenen Sinne spricht also jeder Lieferant seine eigene Sprache. Dadurch kann er, falls erforderlich, der Bibliothek zwar verschiedene Chipversionen vom gleichen Standard anbieten, aber sie kann den Lieferanten nicht wechseln. Der Lieferant verwendet sein proprietäres Datenmodell folglich als Kundenbindungsmassnahme. Bei einem Systemwechsel müssten also sämtliche Etiketten neu beschrieben (nicht ausgetauscht) werden. Da der neue Lieferant die RFID-Chips aber nicht lesen kann, können diese nicht einfach beim Verbuchungsvorgang gelesen und umgeschrieben werden. Sie müssen vielmehr neu initialisiert, d. h. bei jedem Medium der Barcode neu eingelesen werden.

\section{Stellung der Bibliotheken innerhalb der weiteren RFID-Anwendungen}

Mit dem zunehmenden Einsatz von RFID-Systemen hat sich auch die Bedeutung der Bibliotheken innerhalb der RFID-Industrie verändert. Sie sind zu einem Kundensegment geworden, das nachhaltig attraktive Stückzahlen an RFID-Etiketten und Lesegeräten abnimmt. So weckt der Bibliotheksmarkt die Aufmerksamkeit verschiedenster Lieferanten. Dies ist durchaus im Interesse der Bibliotheken. Durch die Konkurrenzsituation bleibt der Markt in Bewegung, Preise sinken und die Versorgung ist sichergestellt.

Die Notwendigkeit der Datenmodellstandardisierung wird auch durch den hohen Anspruch der Bibliotheken an die Lebensdauer der Etiketten gestellt. Bibliotheken »leben « grundsätzlich länger als viele Firmen, denn es sind öffentliche Einrichtungen. Sie müssen also zwingend damit rechnen, dass Etiketten irgend wann nicht mehr funktionieren und/oder Lieferanten im Laufe der Jahre vom Markt verschwinden. Die 
Datenmodellstandardisierung ist also sowohl ein Kosten- wie auch Versorgungsthema, mit mittel- bis langfristigen Auswirkungen.

Bisher stand in der Diskussion über einen Datenmodellstandard so genannte Interlibrary Loan (ILL) im Vordergrund. Er ermöglicht den Austausch von Medien zwischen den Bibliotheken, für den ein standardisiertes Datenmodel die Basis ist. Bei näherer Betrachtung sieht man allerdings, dass der ILL von untergeordneter Bedeutung ist. Der Austausch von Medien bleibt so lange mit traditionellen Mitteln (mit Zetteln und Belegen) durchführbar, wie die Mengen der ausgetauschten Medien auf heutigem überschaubarem Niveau bleiben. Ausserdem wird das gelieferte Buch im ILL nicht in den Medienkreislauf der ausleihenden Bibliothek integriert, sondern stets extra behandelt. Einzig bei der Rückgabe an RFID-Automaten könnten Komplikationen auftreten, wenn ein nicht zur Bibliothek gehörendes Medium mit einem unbekannten Datenmodell angenommen werden müsste. Gegenüber dem ILL ist die Austauschbarkeit der RFID-Komponenten in der Bibliothek, d. h. die Möglichkeit zum Wechsel des Lieferanten, von viel grösserer Bedeutung.

\section{Entwicklung der Standardisierungsarbeiten}

Der erste Ansatz für ein standardisiertes Datenmodell kam 2005 aus den Niederlanden. Dort werden die Nummernkreise für die Medien der nationalen Bibliotheken zentral von der NBD Biblion (Verband der Niederländischen öffentlichen Bibliotheken) vergeben und verwaltet. Dieses Recht wurde auf RFID-Etiketten ausgeweitet und ein eigenes Datenmodell geschaffen. Dieses wurde offen gelegt, aber die NBD behielt die Rechte an den Daten. Sämtliche RFID-Etiketten wurden zentral eingekauft und vorprogrammiert.

Der zentrale Einkauf von RFID-Etiketten wurde nach einiger Zeit aufgegeben. Der Druck, aufgrund der ausländischen Anbieter ständig die Preise anzupassen, wurde so gross, dass sich der Etikettenvertrieb schliesslich nicht mehr lohnte. Heute besteht nur noch der Eigentumsanspruch auf die Mediennummern. Keine Organisation hat dies in ähnlicher Weise durchgesetzt. Umso bemerkenswerter war dieser Sonderweg, da die Niederlande an der Datenmodellstandardisierung in der ISO intensiv mitarbeiteten.

Kurz nach dem Start des NBD-Modells in den Niederlanden wurde das Thema in Dänemark, Deutschland, der Schweiz und Österreich aufgegriffen. In Kopenhagen, am Institut des Dansk Standard (DS) trafen sich erstmals Repräsentanten fast aller RFID-Systemlieferanten an einem runden Tisch, um die technischen Parameter festzulegen, d. h. zu entscheiden welche Daten sinnvoll aufzunehmen und wie diese konkret auf die Chips zu schreiben wären. Im deutschsprachigen Arbeitskreis MSHW (Vertreter der Bibliotheken aus München, Stuttgart, Hamburg, Luzern, Wien) wurden die bibliothekarischen Anforderungen geprüft (welche Daten sinnvoll in den Chip geschrieben werden sollten ). Zusätzlich wurde ein AFI-Wert für die Sicherung der Medien bei der ISO beantragt ${ }^{4}$.

4 Die Klärung und Beantragung des AFI-Wertes (Application Family Identifier) war ein wichtiger Meilenstein. Sie besaß eine ähnliche Bedeutung wie das Datenmodell für die Kompatibilität der RFID-Systeme in Bibliotheken. 
Das Ergebnis war ein dem NBD-Modell ähnliches, aber freies Datenmodell, weil es keine Rechte an Mediennummern festschrieb. Dieses Resultat ist heute als »Dänisches Datenmodell« bekannt. Es wird heute in fast allen Ausschreibungen für RFID-Bibliothekssysteme in Zentral- und Nordeuropa vorgeschrieben. In diversen weiteren Ländern wurden daraufhin Derivate des dänischen Modells entwickelt, die jedoch im Zuge der internationalen Standardisierung auf ISO-Ebene wieder aufgegeben werden. Das Dänische Datenmodell ist als Teil (-3) in ISO 28560 enthalten.

Auch ohne die Festschreibung auf ISO-Ebene hätte sich das Dänische Modell als ein Defacto-Standard durchgesetzt. Es hat in den zurückliegenden Jahren seine Eignung in der Praxis bewiesen. Es sind von den Bibliotheken, welche es bis heute einsetzen, keine Anpassungen oder Erweiterungen gewünscht worden.

\section{Inhaltliche Fragen}

ISO 28560-3 ist inhaltlich in zwei Hauptabschnitte unterteilt:

- Der Mandatory Part enthält die unbedingt erforderlichen Daten, wie z. B. die Mediennummer, Medienpakete und ISIL-Nummer. Er hat einen Mindestumfang von 256 bit.

- Der Optional Part hat eine fast beliebige Grösse und Inhalt. Es nutzt den Speicher über 256 bit hinaus (übliche RFID-Chips haben einen Speicher von 1 bis 2 kbit). In diesem Teil können zum Beispiel Verlage oder Lieferanten logistische Daten hinterlegen. Diese können durchaus verschlüsselt sein.

2007 trat in Kopenhagen die Arbeitsgruppe mit der Bezeichnung ISO-TC46 SC4 WG11 zusammen. Vertreter aus Australien, Dänemark, Deutschland, Finnland, Frankreich, Italien, Neuseeland, Niederlande, Japan, Schweden, Schweiz, Südafrika, Vereinigtes Königreich und den USA beteiligten sich.

Entgegen den ursprünglichen Erwartungen wurde zuerst nicht das bisherige Dänische Datenmodell 1:1 übernommen, sondern versucht, ein vollkommen Neues Modell vorzuschlagen. Es basierte auf einem im Bereich Fluggepäck diskutierten Modell (ISO 15962), welches ein hohes Mass an Flexibilität ermöglichte, da die Felder des Chip-Speichers variabel beschrieben werden konnten. Dieser neue Ansatz kam vielen ISO-Mitgliedern entgegen, da in einigen Ländern noch Uneinigkeit über die eigentlichen Dateninhalte auf dem Chip herrschte. So betrachteten manche Vertreter es als wichtig, dass auch der Buchtitel mit auf dem Chip gespeichert würde. Andere hingegen waren der Meinung, dass diese Inhalte jederzeit vom LMS aus bezogen werden könnten und eine einfache Nummer als Bezug zu den Inhalten ausreichend wäre.

Der neue Vorschlag deckte alle Eventualitäten ab. Es wurden OIDs, so genannte Object Identifier, eingesetzt. Sie verweisen über eine Nummer auf den Speicherbereich im Chip mit der jeweiligen Information. Dieses Modell kann an verschiedene Chipgrössen angepasst werden. Eine wichtige Überlegung für dieses OID-Datenmodell war, dass die Verlage die RFID-Etiketten mit ihren Daten integrieren und diese anschliessend in der Logistikkette, bis in die Bibliothek hinein nutzen können. 
Aufgrund des Einspruches mehrerer Mitglieder wurde jedoch das bisher erarbeitete und bereits in mehreren Ländern etablierte Dänische Datenmodell als eigener Teil 3 mit aufgenommen.

ISO 28560 enthält heute drei Teile mit folgendem Inhalt:

ISO 28560-1 enthält eine Beschreibung vielfältiger, für Bibliotheken denkbarer Datenelemente. Dies sind neben der Mediennummer auch den Titel von Büchern und weitere Daten, welche eventuell offline verfügbar auf dem Chip sein sollten. Aus den Elementen kann für jedes Land ein »Profil« zusammengestellt werden.

ISO 28560 Teil 2 basiert wiederum auf ISO 15962 und den oben genannten OIDs. Er wird in den angelsächsischen Ländern stark propagiert. In diesen Ländern sind bisher vorwiegend proprietäre Datenmodelle im Einsatz, das Dänische Modell kaum verbreitet.

ISO 28560 Teil 3 entspricht zu fast hundert Prozent dem Dänischen Datenmodell. Es ist im Vergleich zum Teil 2 zwar fest kodiert, aber deutlich einfacher strukturiert.

\section{Fazit}

Das Dänische Datenmodell findet sich im Teil 3 von ISO 28560 wieder. Teil 3 wird vorrangig in den zentraleuropäischen und skandinavischen, weniger in den angelsächsischen Ländern eingesetzt. Für die Bibliotheken ist aber mit beiden Teilen, 2 und 3, vorerst eine gute Grundlage geschaffen, auf die sie sich in Ausschreibungen beziehen können. Momentan ist es nicht so wichtig, welcher Standard sich durchsetzt, sondern dass überhaupt ein solcher verwendet wird.

Die Folgen der Standardisierung sind heute erkennbar. Es ist durch die Unabhängigkeit der Bibliotheken von den Lieferanten ein rapider Preisrückgang für die RFID-Etiketten eingetreten. Viele Bibliotheken konnten sich zu einem früheren Zeitpunkt ein RFID-System leisten. Eine weitere Folge ist, dass die Marktentwicklung viel schneller voranschritt, als dies ursprünglich von den Systemanbietern erwartet wurde. Eine erhöhte Transparenz und abnehmende Preise halten sie in Atem. In den angelsächsischen Ländern wird, im Vergleich zu den zentraleuropäischen, der Markt von sehr wenigen Systemlieferanten bestimmt. Dementsprechend ist das Preisniveau dort höher. Dies hat eine Studie 2010 herausgestellt (Mick Fortune ${ }^{5}$ ).

Aus heutiger Sicht macht ein gemeinsamer, zentraler Einkauf von RFID-Etiketten durch Verbände oder gar Verlage, nur wenig Sinn, weil neue Abhängigkeiten entstehen. Die Bibliotheken sind gut beraten, auch weiterhin den Markt scharf zu beobachten.

5 http://www.mickfortune.com/Wordpress/?page_id=201 